Franck BISIAUX ${ }^{1}$

Régis PELTIER ${ }^{2}$

Jean-Claude MuLIELE ${ }^{1}$

1 Projet agroforestier Mampu Fondation Hanns-Seidel Munich, Allemagne

57, avenue des Sénégalais

Gombé, Kinshasa

République démocratique du Congo

2 Cirad

Département Environnements et sociétés

Unité de recherche Ressources

forestières et politiques publiques

TA C-36/D

Campus international de Baillarguet 34398 Montpellier Cedex 5

France

\section{Plantations industrielles} et agroforesterie au service des populations des plateaux Batéké, Mampu, en République démocratique du Congo

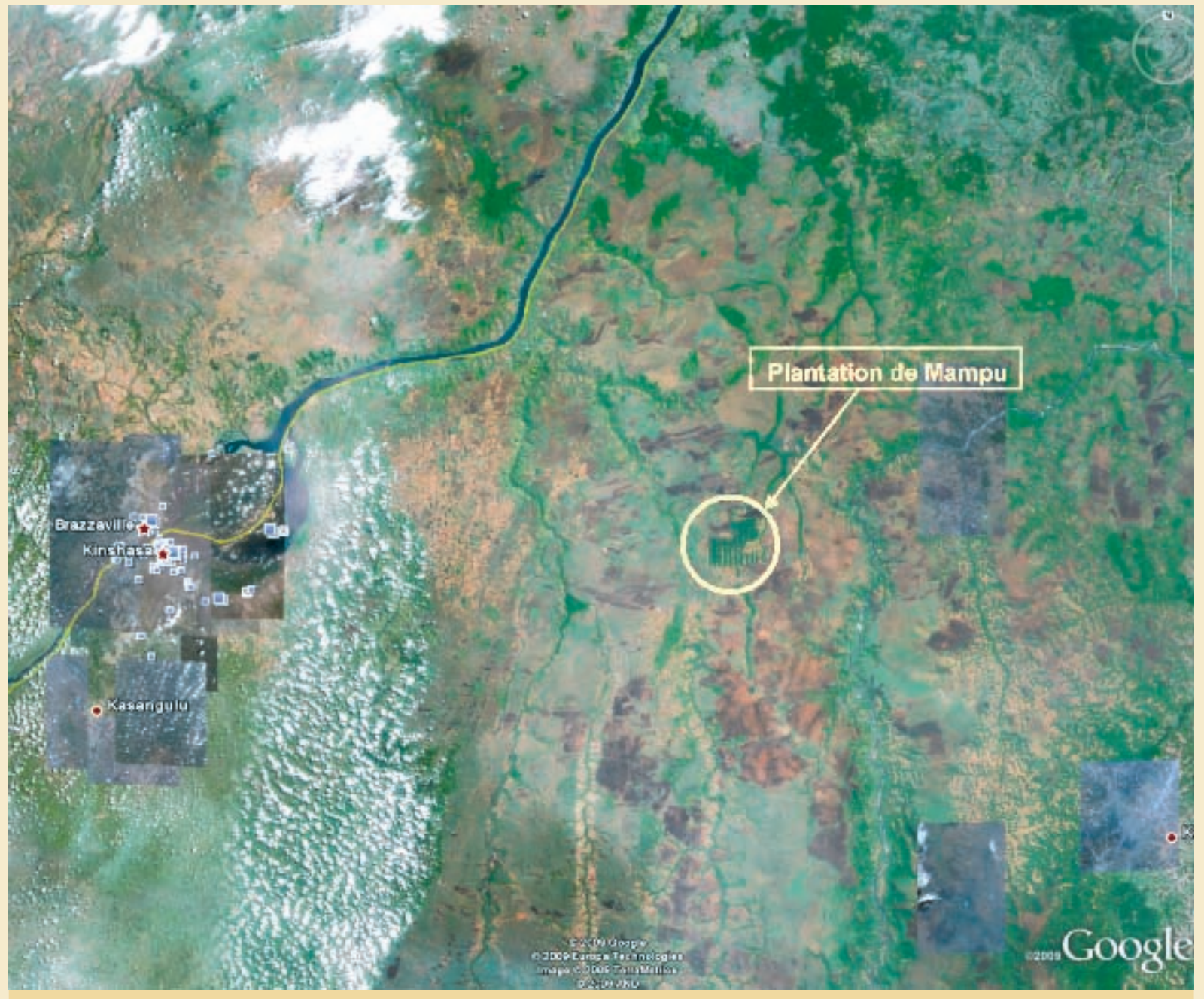

Figure 1.

Image Google Earth localisant le projet Mampu. 


\section{RÉSUMÉ}

\section{PLANTATIONS INDUSTRIELLES ET AGROFORESTERIE AU SERVICE DES POPULATIONS DES PLATEAUX BATÉKÉ, MAMPU, EN RÉPUBLIQUE DÉMOCRATIQUE DU CONGO}

En République démocratique du Congo, Kinshasa, la capitale accueillant les populations issues de l'exode rural et de l'insécurité accrue, concentre environ huit millions d'habitants. La ville est principalement entourée de savanes et d'îlots forestiers dégradés. La consommation en bois énergie est estimée dans une fourchette de trois à six millions de tonnes d'équivalent bois énergie par an (ce qui représenterait 0,6 à 1,2 million de tonnes de charbon de bois par an si tout le bois était carbonisé). Le projet Mampu a été conçu comme la phase pilote d'un projet de reboisement de cent mille hectares sur les sols sableux du plateau Batéké pour faire face à la pénurie de bois et de charbon. Malgré les conflits, environ huit mille hectares de plantations d'Acacia auriculiformis ont été principalement réalisées de 1987 à 1993. À partir de 1998, la plantation de Mampu a été divisée en lots de 25 ha attribués à 320 familles d'agriculteurs. La culture se fait suivant un modèle agroforestier de jachère améliorée, inspiré du modèle traditionnel de culture sur brûlis. La production totale de charbon de ce massifvarie de 8000 à 12000 tonnes annuelles, à laquelle il faut ajouter 10000 tonnes de manioc, 1200 tonnes de maïs et 6 tonnes de miel. Pour le seul charbon, cela correspond à un revenu brut annuel de 2,6 millions de dollars US pour le pays. Au moins un quart revient aux propriétaires agrisylviculteurs. Le succès du projet incite à appliquer ce modèle sur les savanes des plateaux Batéké, en prenant en compte les droits fonciers traditionnels et en poursuivant la diversification et la transformation locale des produits. Cela contribuera à couvrir une part plus importante des besoins urbains en énergie renouvelable, tout en créant des emplois ruraux. Cependant, d'autres modèles de systèmes agroforestiers méritent d'être testés ou développés dans les autres conditions écologiques et socio-économiques du pays, comme la gestion du recrû naturel d'espèces locales à usages multiples appliquée dans le système traditionnel de jachère enrichie (nkunku) du Bas-Congo.

Mots-clés : Acacia auriculiformis, reboisement, charbon de bois, agroforesterie, République démocratique du Congo.

ABSTRACT

\section{INDUSTRIAL PLANTATIONS AND AGROFORESTRY FOR THE BENEFIT OF POPULATIONS ON THE BATÉKÉ AND MAMPU PLATEAUX IN THE DEMOCRATIC REPUBLIC OF THE CONGO}

With people leaving rural areas and fleeing insecurity, Kinshasa, the capital of the Democratic Republic of the Congo, now has some 8 million inhabitants. The city is mainly surrounded by savanna and patches of degraded forest. Estimated fuelwood consumption is in the range of 3 to 6 million tonnes of fuelwood equivalent per year (representing 0.6 to $1.2 \mathrm{MT}$ of charcoal per year if all the wood were charcoaled). The Mampu project was designed as the pilot phase of a reforestation project covering 100000 hectares of sandy soil on the Batéké plateau and aiming to remedy wood and charcoal scarcities. Despite the conflicts, about 8000 hectares of Acacia auriculiformis were planted, mainly from 1987 to 1993 . From about 1998, the Mampu plantation was divided into 25 hectare plots for 320 farming families. Cultivation mainly follows the agroforestry pattern based on improved fallows, which draws on traditional slash-and-burn farming. Total charcoal production from the plantation varies from 8000 to 12000 tonnes per year, in addition to $10000 \mathrm{~T} /$ year of cassava, $1200 \mathrm{~T} /$ year of maize and $6 \mathrm{~T} / \mathrm{year}$ of honey. Gross annual revenue for the country from charcoal alone amounts to 2.6 million US dollars, with owners of the agroforestry plots earning at least a quarter. The success of the project is an incentive to apply the model to the savanna lands on the Batéké plateaux, taking traditional land rights into consideration and continuing activities to diversify and process production locally. This will help to cover a larger share of urban needs for renewable energy while also creating rural employment. However, other agroforestry systems deserve to be tested or developed for different ecological and social or economic conditions across the country, such as management of the natural regrowth of local multiple-use species as applied with the traditional system of fellow enrichment (Nkunku) in the Lower Congo.

Keywords: Acacia auriculiformis, reforestation, charcoal, agroforestry, Democratic Republic of the Congo.

\section{RESUMEN}

\section{PLANTACIONES INDUSTRIALES Y AGROFORESTERÍA AL SERVICIO DE LOS HABITANTES DE LAS MESETAS BATEKE EN LA REPÚBLICA DEMOCRÁTICA DEL CONGO}

En Kinshasa, capital de la República Democrática del Congo que absorbe la población del éxodo rural y de la creciente inseguridad, se concentran unos ocho millones de habitantes. La ciudad está principalmente rodeada por sabanas e islotes forestales degradados. El consumo dendroenergético se estima en una horquilla de entre tres y seis millones de toneladas (MT) de equivalente en leña por año (lo que supondría de 0,6 a 1,2 MT de carbón vegetal/año, si toda la leña fuera carbonizada). El proyecto Mampu se concibió como fase piloto de un proyecto de reforestación de cien mil hectáreas en los suelos arenosos de la meseta Bateke para combatir la escasez de madera y carbón. A pesar de los conflictos, se pudieron plantar unas ocho mil hectáreas de Acacia auriculiformis, principalmente entre 1987 y 1993. A partir de 1998, la plantación de Mampu fue dividida en lotes de 25 ha atribuidos a 320 familias de agricultores. Los cultivos se realizan siguiendo un modelo agroforestal de barbecho mejorado, inspirado del cultivo tradicional de quema. La producción total de carbón de esta masa forestal varía entre 8000 y 12000 toneladas anuales (T/año), a la que hay que añadir 10000 T/año de mandioca, 1200 T/año de maíz y 6 T/año de miel. Únicamente el carbón, ya representa unos ingresos anuales de 2,6 millones de dólares US para el país. Los propietarios agrosilvicultores reciben, al menos, una cuarta parte. El éxito del proyecto incita a aplicar este modelo en las sabanas de las mesetas Bateke, teniendo en cuenta los derechos consuetudinarios sobre la tierra y continuando con la diversificación y transformación local de los productos. Esto contribuirá a cubrir una parte cada vez mayor de las necesidades urbanas de energía renovable, al tiempo que se crean empleos rurales. Sin embargo, merece la pena probar o desarrollar otros modelos de sistemas agroforestales adaptados a otras condiciones ecológicas o socioeconómicas del país, como el manejo de rebrotes naturales de especies locales de usos múltiples aplicado al sistema tradicional de barbechos enriquecidos (Nkunku) del Bajo Congo.

Palabras clave: Acacia auriculiformis, reforestación, carbón vegetal, agroforestería, República Democrática del Congo. 


\section{Le contexte}

La République démocratique du Congo ( $R d c)$ est le troisième plus vaste pays d'Afrique et le plus peuplé d'Afrique centrale. Le nord du pays est un des plus grands massifs de forêt équatoriale au monde. L'est du pays borde le Grand Rift est-africain, domaine des montagnes, des collines, des grands lacs et des volcans. Le sud et le centre, en grande partie couverts par des savanes arborées, forment un plateau s'élevant vers le sud. Au sud-ouest, la forêt du Mayombe et au sud-est la forêt claire de type Miombo forment des massifs forestiers typiques parmi les plus soumis à la pression anthropique.

Comparativement à sa taille, le Congo-Kinshasa est peu peuplé, la population se concentre sur les plateaux, dans la savane près des fleuves et des lacs. Le nord et le centre du pays, domaine de la forêt, sont pratiquement vides. Aggravé par l'insécurité et les guerres civiles, l'exode rural a gonflé les villes et en particulier Kinshasa (population estimée à huit millions d'habitants). Cette ville a des besoins énormes en énergie. Avec l'hypothèse couramment admise dans les villes d'Afrique de l'Ouest à forte pénurie (par exemple Niamey au Niger) que chaque habitant consomme pour son énergie domestique (cuisson des aliments et marginalement chauffage) un kilogramme d'équivalent bois (éq. bois) par habitant et par jour (BERTRAnd, 1991), Kinshasa consommerait près de 3 millions de tonnes d'équivalent bois par an, ou l'équivalent d'environ 600000 tonnes de charbon par an. D'après GAzULL (2009), cette consommation serait plus du double dans des capitales telles que Bamako (2,5 kg éq. bois/hab./j en 2004) et Tananarive (2,8 kg éq. bois/hab./j en 2009), l'augmentation récente étant due au passage du bois au charbon de bois.

Or, dans un rayon de $150 \mathrm{~km}$ autour de Kinshasa, il n'y a que très peu de massifs forestiers susceptibles de fournir ce bois énergie. La

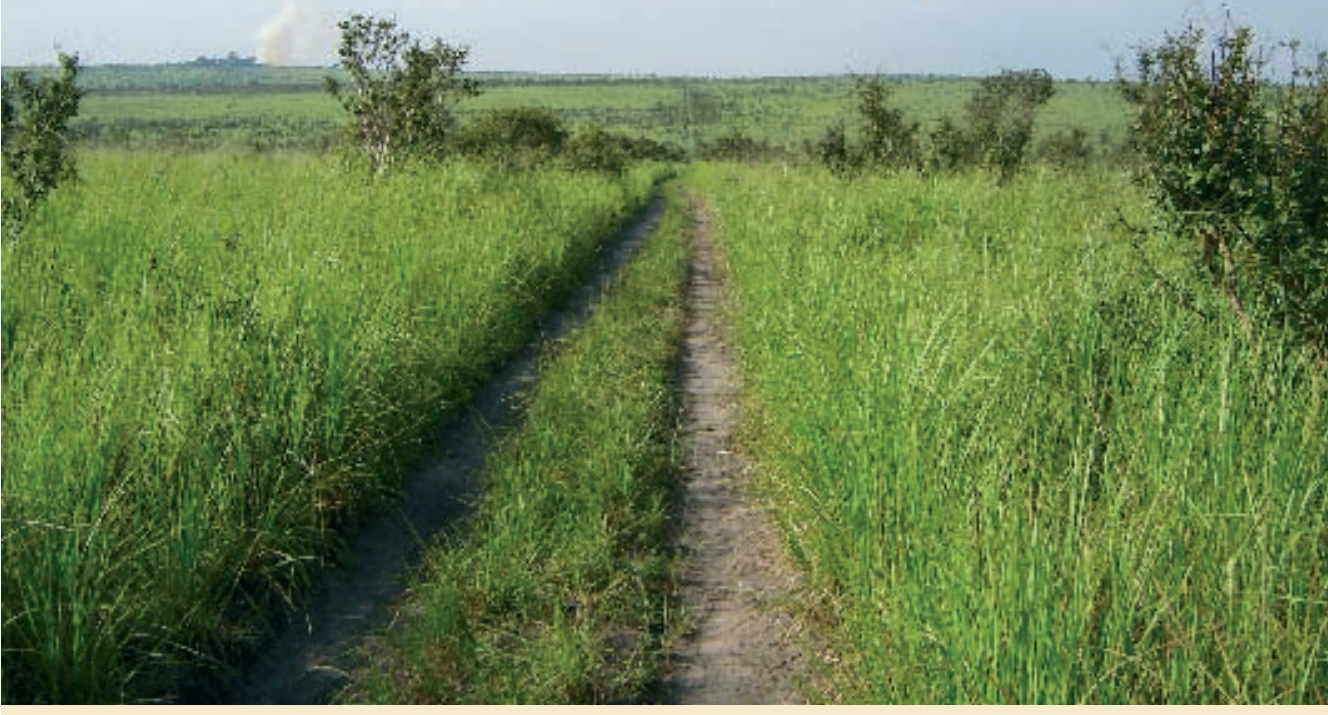

Photo 1.

Savane arbustive, caractérisée par Hymenocardia acida.

Photo R. Peltier.

majorité de cette zone est occupée par le plateau Batéké. Son relief (700 m d'altitude moyenne) est faiblement ondulé à l'exception des vallées assez larges de la Vue, la Mwana et la Mbali supérieure (550 à $630 \mathrm{~m}$ d'altitude) et des vallées plus encaissées de la Lufimi, la Mbali inférieure et du Kwango (200 à 350 m d'altitude) (LADMIRANT, 1964). Les flancs de ces vallées et de leurs affluents portaient autrefois des forêts denses (DuvigneAud, 1949), aujourd'hui plus ou moins dégradées par l'agriculture itinérante (beaucoup d'auteurs qualifient ces forêts de " galeries ", bien qu'elles s'étendent bien au-delà du lit majeur des cours d'eau, comme c'est le cas en zone soudanienne). Le climat est tropical chaud avec une saison sèche de quatre mois de juin à septembre. Les températures annuelles moyennes oscillent autour de $25^{\circ} \mathrm{C}$. Les sols sont surtout sablonneux, acides, chimiquement pauvres et possèdent une très faible capacité de rétention hydrique (KoY KASANGO, 2005). La kaolinite est le matériau le plus important de la fraction argileuse. Le $\mathrm{pH}-\mathrm{H}_{2} \mathrm{O}$ est en général inférieur à 5,5 et varie avec la teneur en matière organique.

La savane est la formation naturelle dominante avec quelques galeries forestières (ROBYNS, 1948) :
- savanes herbeuses à Loudetia, caractérisées par Loudetia arundinacea et Ctenium newtonii ;

- savanes arbustives dégradées à Digitaria, caractérisées par Digitaria uniglumis, Hyparrhenia diplandra et Hymenocardia acida ;

- savanes arbustives, caractérisées par Hymenocardia acida et parfois parsemées d'arbres, notamment Erythrina abyssinica et Cussonia angolensis (photo 1) ;

- galeries forestières, du type périguinéen, se trouvant sur les pentes raides et les vallées des rivières Mbali, Mwana, Kwango et Lufumi (photos 2).

La zone du plateau Batéké est située dans la province administrative de Kinshasa. Historiquement, cette zone était peu densément peuplée par l'ethnie Téké : environ trois habitants au kilomètre carré. L'autorité traditionnelle est exercée par les chefs coutumiers dont le rôle, en droit moderne, n'est pas clairement précisé en matière judiciaire et foncière. L'ordre public, l'hygiène, la santé, l'éducation, les communications sont du ressort des autorités territoriales.

Les activités économiques sur le plateau sont essentiellement orientées vers l'agriculture itinérante. L'infrastructure routière y est peu développée. 


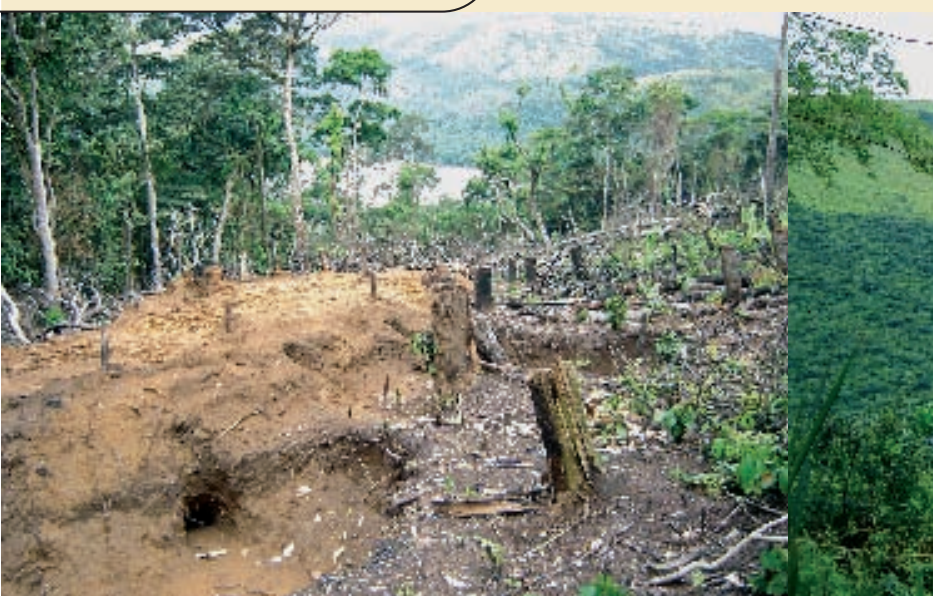

Forêt des versants du plateau Batéké en cours de défrichement pour la production de charbon et la mise en culture agricole. Photo R. Peltier.

\section{Mampu, projet de reboisement industriel lors des conflits}

En 1984, devant la pénurie de bois énergie à Kinshasa, le projet Mampu fut conçu comme la phase pilote d'un projet de boisement d'importance, d'environ 100000 ha, qui devait poursuivre un objectif annuel de production de 126000 t de charbon, à partir de l'an 2000 (DUCENNE, 2009). Cet objectif de production devait correspondre à 30-40\% de la demande en charbon de bois totale estimée de la capitale (environ 400000 t/an, à l'époque). La phase pilote (8 000 ha) a été mise en œuvre par la société $\mathrm{Hva}^{1}$ sous le contrôle de la société Zte ${ }^{2}$. Le financement de cette phase pilote s'appuya sur les ressources du Fonds européen de développement (Fed), de l'ordre de 8500000 Ecu.

Ce projet est situé sur le plateau Batéké $\left(4^{\circ} 20 \mathrm{~S}, 16^{\circ} 18 \mathrm{E}\right)$, à une altitude de $670-720 \mathrm{~m}$. Il se trouve à $150 \mathrm{~km}$ à l'est de Kinshasa (dont 26 km de piste sableuse depuis Mbankana).

L'emplacement a été choisi à proximité immédiate du centre de recherche de Kinzono, créé en 1976 sur financements nationaux zaïrois et avec l'aide de la coopération belge ( $M$. GERKENS, com. pers.). Ayant testé plus de quatre-vingts espèces locales et exotiques, ce centre avait montré l'ex- cellente croissance de l'espèce Acacia auriculiformis. Par ailleurs, les conflits fonciers avec les autorités traditionnelles téké y semblaient modérés.

Entre 1987 et 1993, la société Hva a boisé 7262 ha de savane dégradée (photo 3), principalement à l'aide d'Acacia auriculiformis (plus de $95 \%$ de la surface plantée) et d'eucalyptus dans une mesure très marginale. Pour cela, une pépinière de 6 ha ayant une capacité de production annuelle de 4 millions de plants forestiers fut aménagée (surdimensionnée en vue d'une extension souhaitée du projet).

Suite aux événements et aux pillages de 1991, la société Hva abandonna les travaux en mars 1992 Soucieux de l'avenir du projet, le gouvernement congolais confia à la Fhs ${ }^{3}$ la mission de maintenir les infrastructures du projet jusqu'en décembre 1993. De janvier 1994 à mai 1995, la Fhs et le Cadim ${ }^{4}$ se virent confier le mandat d'assurer la protection de la concession. Au terme de cette période, un protocole d'accord a été signé entre le gouvernement congolais, la Fhs et l'Union européenne (UE), cette dernière accordant un crédit de maintenance des plantations à la Fhs (DuCEnNe, 2009).

Depuis 2003 et la reprise de la coopération de l'UE, deux nouveaux projets européens ont appuyé le développement de Mampu (contribution à la relance de la production agricole par la promotion de l'agroforesterie et sa diffusion en milieu villageois sur le plateau Batéké).

\section{À partir de 1994, une évolution délibérée vers l'agroforesterie}

\section{Un modèle de jachère améliorée inspiré \\ du système traditionnel de culture sur brûlis}

À partir des années 1998, la plantation de Mampu fut divisée en lots de 25 ha qui ont été attribués à des agriculteurs. Ceux-ci devaient gérer leur plantation, avec l'encadrement technique de la Fhs, suivant un modèle agroforestier inspiré du modèle traditionnel de culture sur brûlis.

En effet, si les termes « système agroforestier » ont un sens très large d'association entre arbre, culture et élevage, dans l'espace et/ou dans le temps (COMBE, BudowsKı, 1979 ; NAIR, 1985 ; BAUMER, WOOD, 1986 ; LUNDGREN, 1987), on peut parler de " système agroforestier séquentiel " quand il s'agit d'alternance dans le temps, sur une même parcelle, entre culture et forêt. C'est le cas pour l'amélioration de l'agriculture itinérante par la jachère améliorée improved fallow - (TORQUEBIAU, 1990). La parcelle n'est pas abandonnée après les quelques saisons de culture, mais enrichie de ligneux utiles. Il est possible d'ensemencer la jachère soit par plantation, soit par régénération naturelle à partir
1 Hollandaise Agro-Industries.
2 Zaïre Trading Engineering.

\footnotetext{
3 Fondation Hanns-Seidel.

${ }^{4}$ Centre d'appui au développement intégré de Mbankana.
} 
d'arbres fixateurs d'azote, qui rétablissent un sol de bonne qualité plus vite que les espèces spontanées.

C'est le cas à Mampu où, en principe, chaque année, l'agrisylviculteur (agriculteur pratiquant l'agroforesterie) exploite une parcelle d'environ 2 ha, transforme le bois en charbon, brûle les résidus en début de pluie et met en place sa culture mélangée de maïs et de manioc. La surface de 2 ha est un maximum théorique, compte tenu des parefeux et des pistes, mais en réalité la Fsh a limité à un hectare et demi, pour tenir compte des zones vides et pour éviter un pillage du capital bois. Il faut noter que $A$. auriculiformis ne rejette pas de souche, après la coupe. Le passage superficiel du feu lève la dormance des graines d'acacia qui germent en grand nombre (photos 4). Lors des sarclages de ses cultures, l'agrisylviculteur les préserve sur les lignes qui joignent les souches mortes. Au besoin, il peut regarnir les zones où les semis naturelssont trop rares. Quatre mois après le feu, à la récolte du maïs, les acacias ont environ un mètre de haut ; 18 mois après le feu, à la récolte du manioc, les acacias ont environ trois mètres de hauteur (photo 5). Ce gaulis obtenu par régénération naturelle assistée (Rna) peut se développer sans autre intervention humaine, en dehors d'une éclaircie (dans les zones trop denses ne sont obtenues que des gaulettes nombreuses mais trop fines pour être carbonisées), de la protection contre le feu et de l'élimination de quelques espèces arborées envahissantes. Il peut s'agir, par exemple, d'Anthocleista schweinfurthii (mupuku-puku en kikongo), que les agriculteurs conservent jusqu'à un certain diamètre pour les abattre et cueillir des champignons sur le bois en cours de putréfaction. Douze ans plus tard, un retour est possible pour exploiter à nouveau la parcelle.

Réalisé en 2008 dans des parcelles plantées de dix-neuf ans, un inventaire a fourni des volumes variant entre 190 à $340 \mathrm{~m}^{3} / \mathrm{ha}$, soit un accroissement annuel moyen de

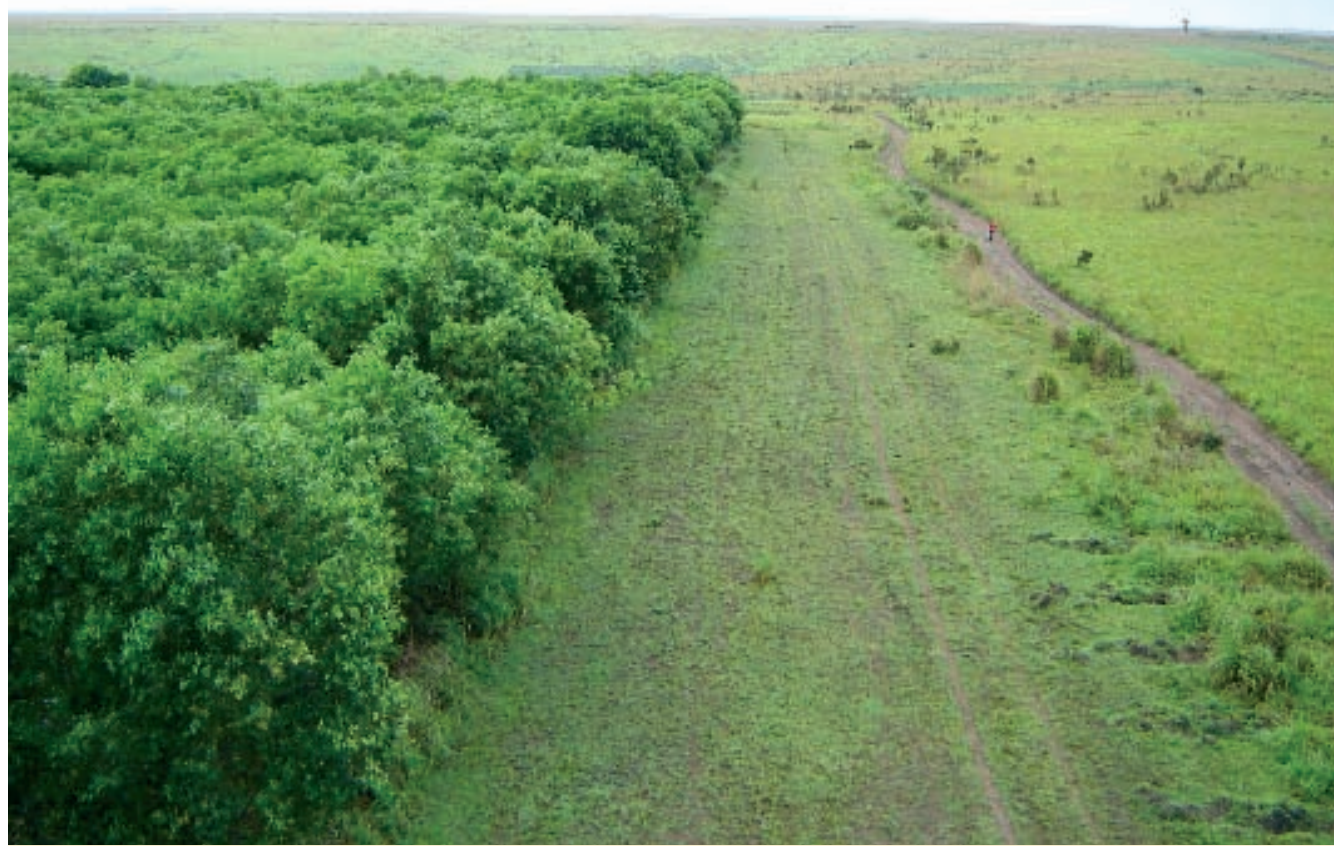

Photo 3.

Entre 1987 et 1993, la société Hva a boisé 7262 ha de savane dégradée, principalement à l'aide d'Acacia auriculiformis.

Photo R. Peltier.

10 à $18 \mathrm{~m}^{3} / \mathrm{h}$ a et une moyenne de $12 \mathrm{~m}^{3} /$ ha. En principe, une parcelle de douze ans pourrait contenir $144 \mathrm{~m}^{3} /$ ha, soit environ $120 \mathrm{t}$ de bois sec à l'air, qui donnerait $24 \mathrm{t} / \mathrm{ha}$ de charbon (avec un rendement de carbonisation de $20 \%$ ) ou quatre cents sacs de $60 \mathrm{~kg}$ de charbon par hectare.

\section{Des charbonniers de plus en plus performants}

La carbonisation est de mieux en mieux maitrisée par les agrisylviculteurs de Mampu. Une meule de trente stères, soit environ $24 \mathrm{t}$ de bois sec à l'air, donne en moyenne 80 à 90 sacs de $60 \mathrm{~kg}$, soit 5,1 t, ce qui correspond à un rendement légèrement supérieur à $20 \%$ du poids sec à l'air (photos 6). Ce rendement est satisfaisant par rapport aux rendements maximaux que l'on trouve dans la littérature pour la carbonisation en meule ( $23 \%$ d'après BRIANE et DoAT, 1985) et les accidents (incendie de la meule, brûlures des charbonniers) se font heureusement de plus en plus rares.

\section{Des agriculteurs très productifs, malgré la pauvreté initiale du milieu}

Alors que l'agriculture traditionnelle sur brûlis de type téké ne s'intéresse qu'aux îlots de forêt dense et considère que les sols de savane sont trop pauvres pour être valorisés, c'est au contraire sur ces sols qu'ont été installées les plantations, puis le système agroforestier.

La présence d'une légumineuse arborée (l'acacia), fixatrice d'azote, dans la jachère améliore les paramètres de fertilité du sol sous plantations d'acacia, tels que le taux de matière organique (qui augmente de $1,9 \%$ après 17 ans), le taux d'azote, la Cec (capacité d'échange des cations) et la somme des bases qui augmentent, et le rapport $\mathrm{C} / \mathrm{N}$ (carbone par rapport à azote) qui diminue avec l'âge de la plantation. Ainsi, la culture traditionnelle associée de maïs (récolte à 3-4 mois) et de manioc (récolte à 18 mois) devient possible après exploitation. 


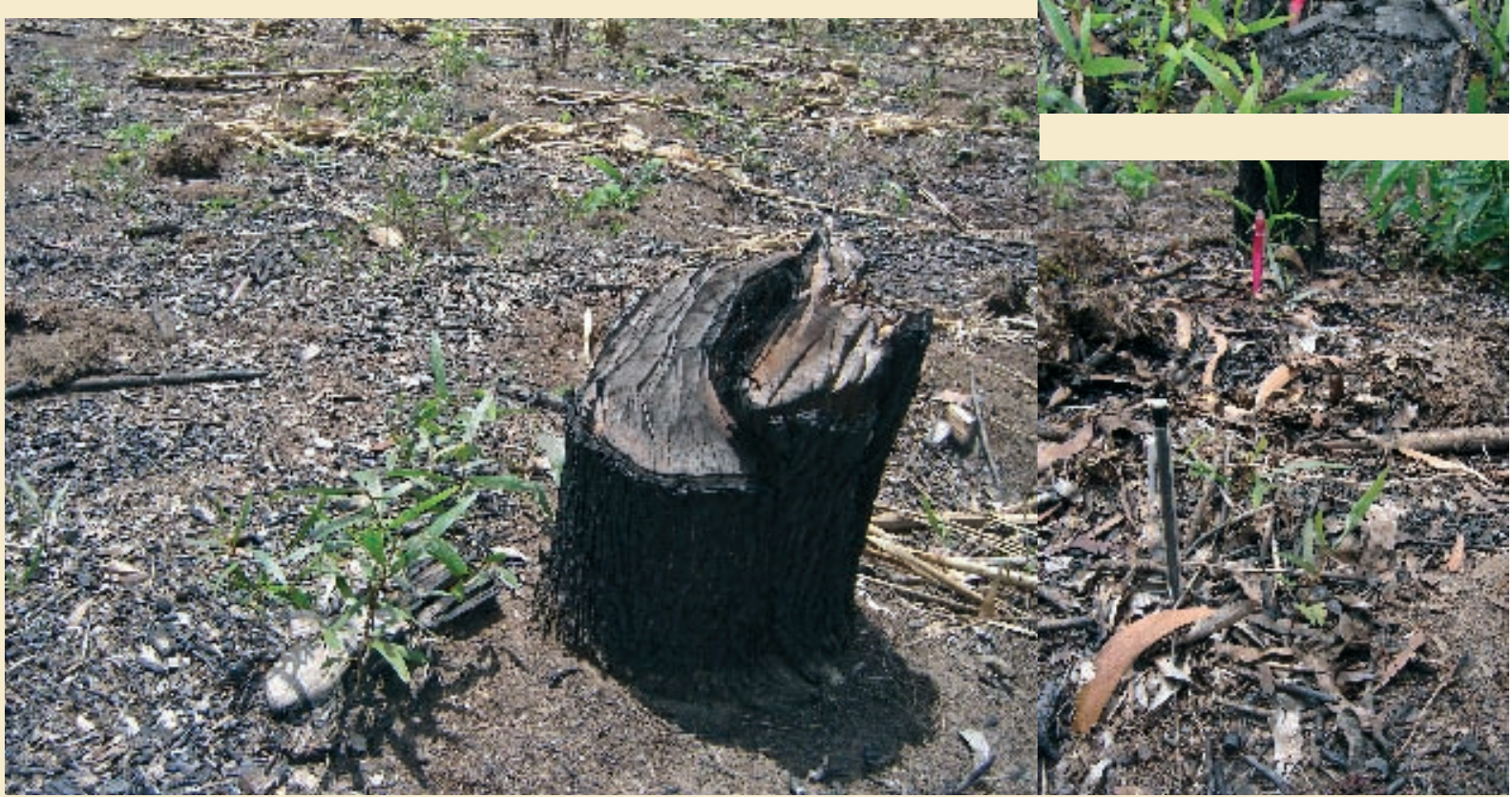

Photos 4.

A. auriculiformis ne rejette pas de souche, après la coupe. Le passage superficiel du feu lève la dormance des graines d'acacia qui germent en grand nombre. Photo R. Peltier.

Compte tenu des médiocres conditions matérielles des agrisylviculteurs de Mampu (absence d'engrais, de chaulage, d'herbicides et de labour ou broyage mécanique), l'amélioration des sols est grandement facilitée par un brûlis des rémanents aériens (feuilles, brindilles), en début des pluies et juste avant le semis du maïs. Ce brûlis permet d'accéder aisément, d'éliminer les adventices et les parasites, de remonter le $\mathrm{pH}$ et de libérer une partie des éléments minéraux stockés dans cette biomasse. En conditions de sol humide, l'essentiel de la matière organique du sol (humus et racines) n'est pas dégradé par le feu. En outre, avec l'appui du projet, les agrisylviculteurs utilisent en très grande majorité des semences de maïs et des boutures de manioc améliorées, et en particulier des clones de manioc résistant à la mosaïque. De ce fait, un agrisylviculteur, sur un hectare et demi, peut récolter en une année 1,5 ha de maïs et 1,5 ha de manioc (photos 7). La production totale du périmètre, pour 320 exploitations de 25 ha, peut ainsi être estimée à environ 10000 t/an de manioc et $750 \mathrm{t} / \mathrm{an}$ de maïs (chiffre majoré à 1200 t/an, grâce à des cultures sur pare-feux ou autres).

\section{Un souci de diversification et de valorisation des produits du système agroforestier}

Depuis 2004, l'équipe du projet encourage la diversification et la transformation sur place des produits du périmètre. C'est ainsi que l'apiculture a été introduite pour valoriser le fort potentiel mellifère des acacias. La production de miel du massif est collectée par le Regroupement des agriculteurs de Mampu qui la vend au Cadim. La production a rapidement augmenté de 3500 kg en 2005 à 8000 kg en 2007, pour se stabiliser autour de $6000 \mathrm{~kg}$ en 2008. Le miel est vendu 2,1 dollars américains par kilogramme (monnaie utilisée localement), soit un revenu brut annuel de 12600 dollars US. Alors que le manioc était vendu en tubercules (très coûteux à transporter en raison de leur forte teneur en eau), le séchage sous forme de cossettes ( $3-5 \mathrm{~cm}$ de diamètre) ou de micro-cossettes (2-3 mm de diamètre) a été encouragé. Les micro-cossettes de manioc sont fabriquées sur place, après épluchage manuel, râpage mécanique, rouissage et séchage des tubercules. Le rendement (micro-cossettes sèches/ tubercules frais) est estimé à $34 \%$. Les micro-cossettes sont vendues un dollar le kilogramme, en sacs de $25 \mathrm{~kg}$, par le Groupement des producteurs de manioc et le Cadim, mais ne représentent encore qu'une minorité de la production de manioc.

En principe, les meilleurs agrisylviculteurs de Mampu ont des revenus beaucoup plus élevés que la moyenne des agriculteurs de la zone. La coupe de $1,5 \mathrm{ha} / \mathrm{an}$ permet la fabrication de 600 sacs de charbon de $60 \mathrm{~kg}, 4,5$ dollars par sac revenant au propriétaire, soit de l'ordre de 2700 dollars par an (Sur un sac vendu 18 dollars à Kinshasa, soit 13500 francs congolais, une première estimation montre que 9 dollars vont à la main-d'œuvre et 4,5 dollars aux transports et taxes.) À ces revenus, s'ajoutent les productions agricoles de maïs (3,75 t par agrisylviculteur et par an) et manioc (30 t par agrisylviculteur et par an). Pour certains propriétaires, récolter le miel, transformer leur manioc en micro-cossettes ou réaliser les coupes, la carbonisation et la vente du charbon par eux-mêmes, procure des revenus complémentaires. Certains ménages peuvent donc gagner annuellement près de 4000 dollars US, soit l'équivalent du salaire d'un cadre. 


\section{Une production de charbon avec retour sur investissement}

Au sein du massif de 8000 ha, le calcul de la production totale de charbon varie annuellement, suivant les sources, de 8000 à 12000 t ou 5 t / ha/an de bois (6 à $7 \mathrm{~m}^{3} / \mathrm{ha} / \mathrm{an}$ ), avec un rendement de carbonisation de $20 \%$. Mais cette production paraît faible, rapportée aux 8000 ha du massif et aux chiffres donnés dans la littérature sur l'accroissement de $A$. auriculiformis, dans des conditions écologiques similaires, souvent supérieurs à $15 \mathrm{~m}^{3} / \mathrm{ha} / \mathrm{an}$ (BERNHARD-REVERSAT et al., 1993). La prudence de la Fhs qui ne laisse pas exploiter toute la production annuelle explique ces chiffres. Quoi qu'il en soit, cette production correspond au moins à 130000 sacs de charbon et à un revenu brut de 2,6 millions de dollars par an pour le pays, dont au moins le quart revient aux propriétaires agrisylviculteurs. Par un calcul économique très simple, il apparaît que quatre années de production de charbon remboursent les investissements initiaux faits par l'UE.

\section{Un écosystème forestier qui se reconstitue}

Bien qu'il n'y ait pas eu de mesure scientifique de l'évolution de la biodiversité, la perception des agrisylviculteurs et de la plupart des visiteurs est qu'un écosystème forestier se reconstitue assez rapidement sur le périmètre du projet, en lieu et place de l'ancien écosystème de savane. De nombreuses espèces végétales et animales qui n'étaient présentes que dans les îlots forestiers se retrouvent aujourd'hui dans la majorité des parcelles. C'est le cas, par exemple, pour les ignames sauvages (photo 8) et pour de nombreuses autres lianes et espèces arborées pionnières comme Anthocleista schweinfurthii. Cela incite les habitants à y développer des pratiques de cueillette (champignons, chenilles, tubercules, etc.) et de chasse (rongeurs, reptiles, céphalophes, etc.), autrefois limitées aux zones forestières.

\section{Discussion : durabilité ?}

\section{Paramètres de production et de fertilité}

Subsistent encore diverses incertitudes en ce qui concerne la surface exploitée chaque année, la croissance des plantations, avant et après régénération naturelle assistée, les rendements réels à la carbonisation et l'évolution à long terme de la fertilité des différents types de sol (en raison des exportations de bois et de produits agricoles, non compensées par des apports d'engrais).

Les cadres du projet et certains agrisylviculteurs estiment qu'il est très important de brûler les résidus par un feu courant de début de saison des pluies (qui carbonise plus qu'il ne consume) ainsi que de fabriquer le charbon sur les parcelles elles-mêmes. Cela permet de laisser au sol les cendres et les résidus de charbon qui se dégradent très lentement et augmentent la capacité d'échange des sols (ils l'observent, en particulier, par la taille des cultures et des arbres sur les anciennes meules). Cet aspect mérite d'être chiffré pour conseiller aux cultivateurs d'épandre ces résidus sur l'ensemble des parcelles. Par manque de moyens de transport, la méthode la plus facile consiste à faire des fours plus petits et mieux répartis dans l'espace cultivable. Également, il s'agit de suivre la composition biologique du sol (microfaune, macrofaune, microflore), la comparer à celle de la savane et d'évaluer l'impact du brûlis des résidus.

Les observations des agrisylviculteurs et des techniciens de terrain, qui allaient contre l'avis des scientifiques des années 1990 , majoritairement opposés au brûlis des rémanents, rencontrent celles d'une nouvelle génération de chercheurs. En effet, le bénéfice des résidus organiques est de courte durée sous les tropiques (JENKINSON, AyAnABA, 1977) ; c'est pourquoi de plus en plus de scientifiques préconisent la gestion du charbon organique (référencé sous le nom de "Bio-Char ») dans les sols tropicaux,

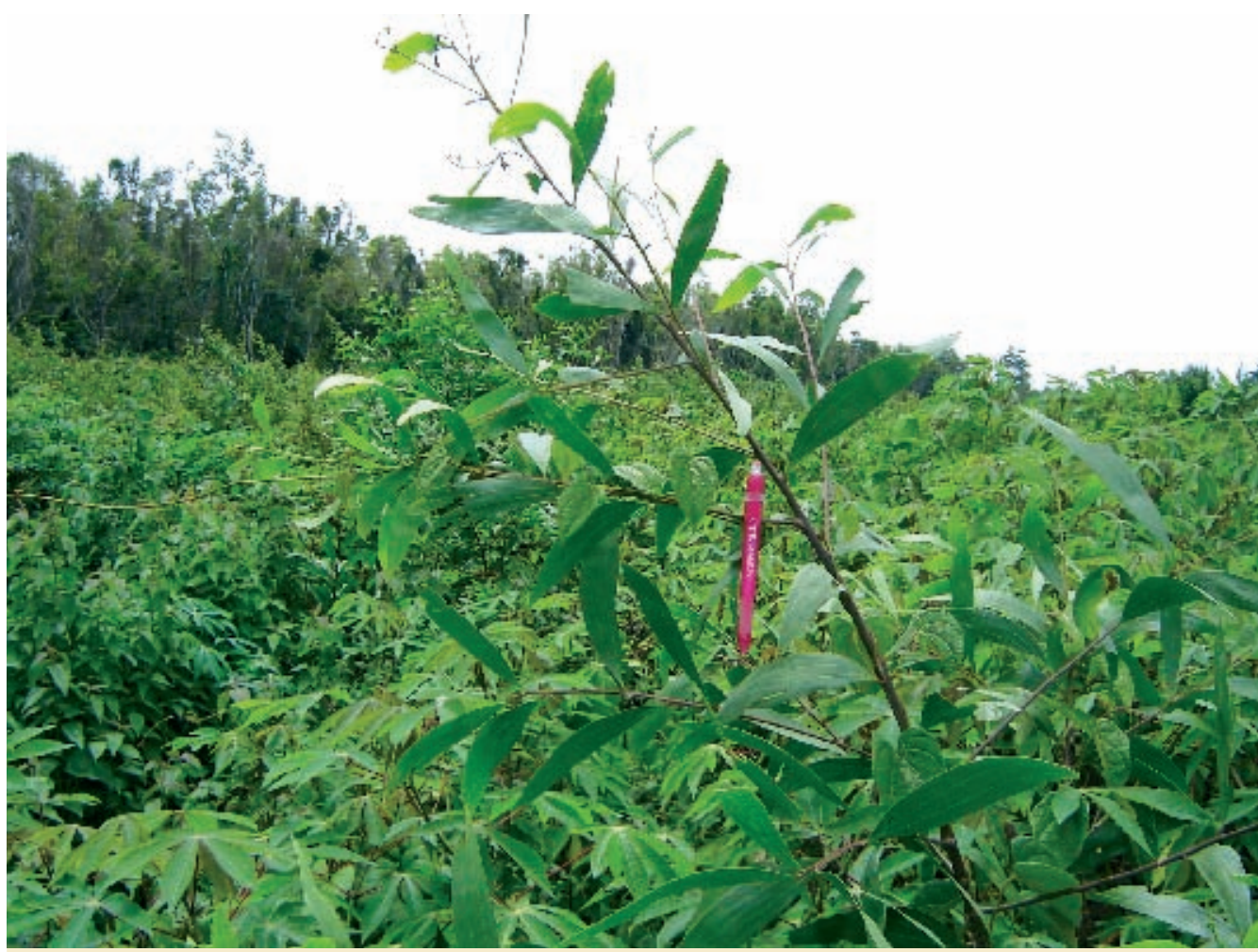

Photo 5.

Dix-huit mois après le feu, à la récolte du manioc, les acacias ont environ $3 \mathrm{~m}$ de hauteur.

Photo R. Peltier. 
afin d'en maintenir la fertilité (GLASER et al., 2002). Cela a été confirmé par l'étude de sols du Bassin amazonien, autrefois occupé par des campements d'Amérindiens
(Terra Preta de Indios) qui ont conservé leur fertilité depuis cinq à vingt-cinq siècles, grâce à leur teneur en charbon de bois (LEHMANN et al., 2003). C'est ainsi que LEHMANN et
RoNdon (2006) préconisent la réhabilitation du système de culture sur brûlis ou abattis-brûlis (slash-andburn) sous le nom réhabilité de culture sur charbon (slash-and-char).

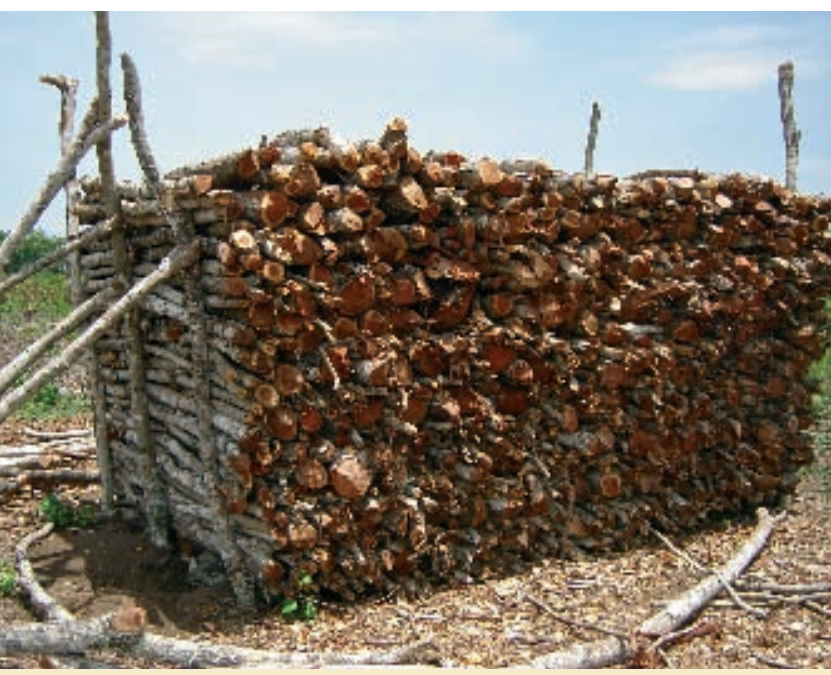

Photos 6.

Des charbonniers de plus

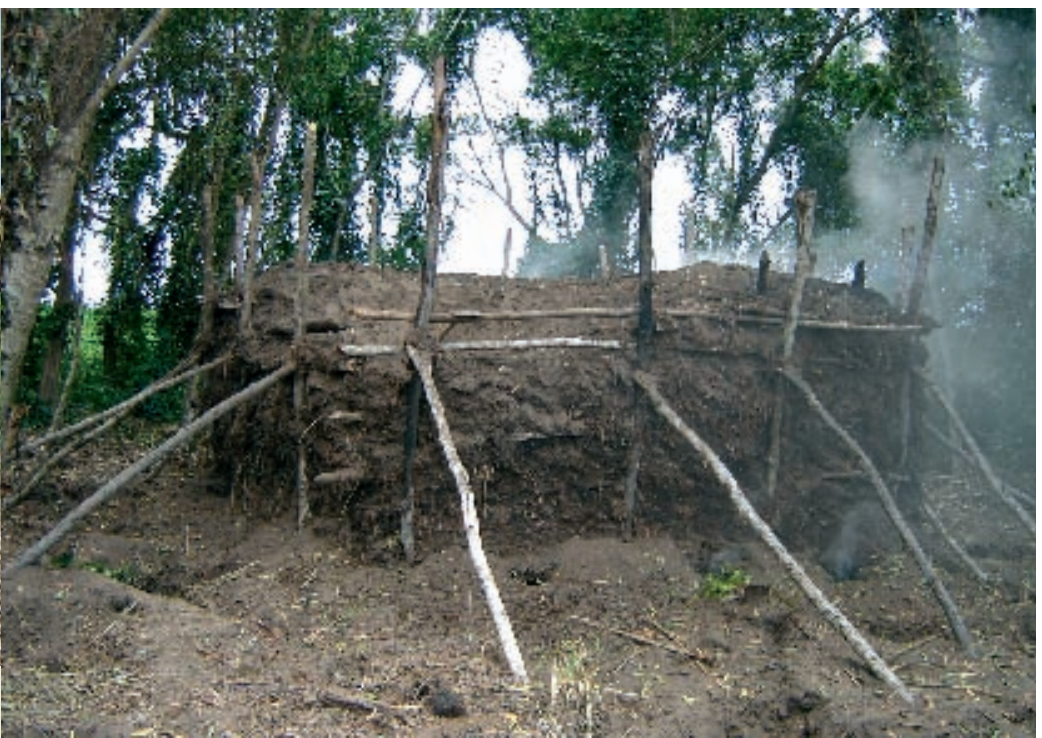

en plus performants.

Photo R. Peltier.

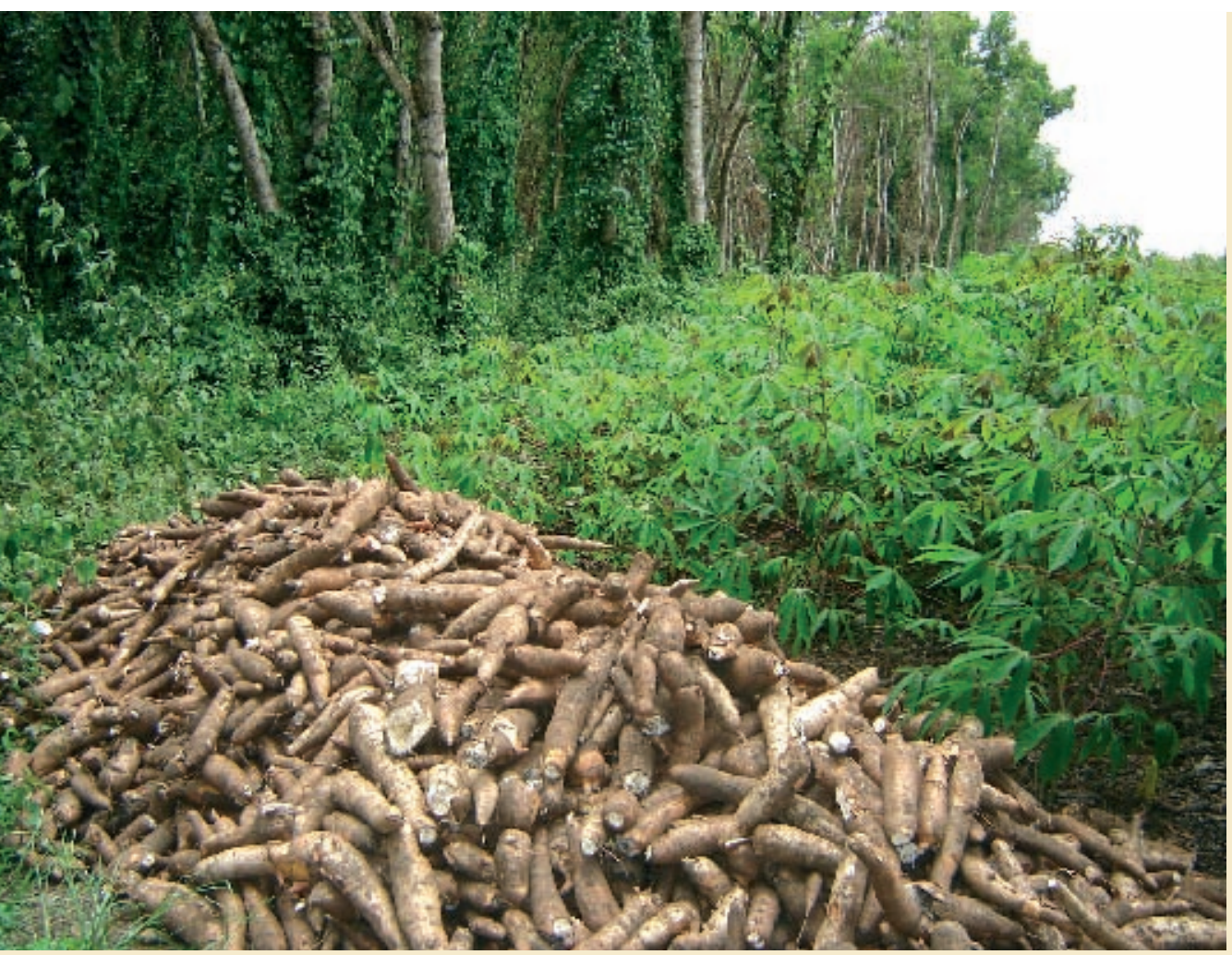

Photos 7.

Récolte de manioc, après culture sur brûlis de plantation d'acacia et transformation en cossettes. Photo R. Peltier.

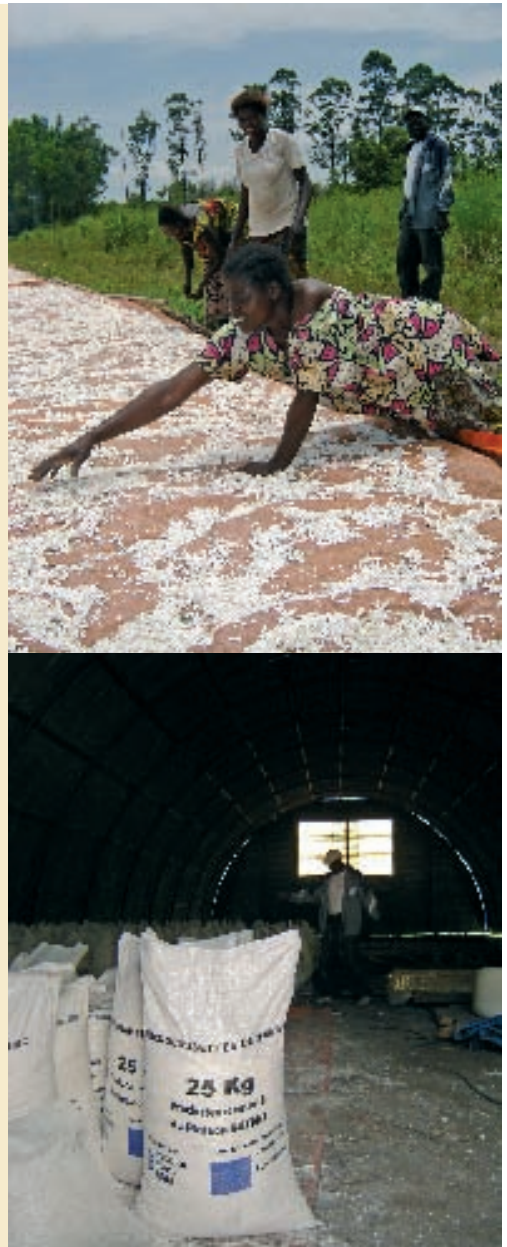




\section{Organisation sociale du site de Mampu}

Spécialiste des aspects sociaux et politiques, la Fhs s'intéresse à l'évolution des organisations paysannes et à leur capacité à gérer cette nouvelle cité de Mampu et ses infrastructures rurales (pistes, pare-feux), artisanales (ateliers à micro-cossettes de manioc et à affinage du miel) et urbaines (marché, école, dispensaire, etc.), accueillant près de cinq mille habitants. En effet, le statut de Mampu reste pour l'instant intermédiaire entre celui de chantier (administré par un projet) et celui de ville (administrée par l'État). La Fsh étudie également l'évolution du foncier rural (transmission, vente, location, concentration), des filières de charbon et de produits agricoles (organisation, prise de contrôle, taxation formelle et informelle). Demeurent enfin les difficiles problèmes de l'insécurité grandissante et de la diversification des produits autoconsommés ou vendus : fruits, plants ou boutures d'arbres fruitiers, champignons, chenilles, bois non carbonisé, etc.

\section{Incertitudes sur les prochains cycles de plantation/production (rotations) d'acacias obtenus par semis naturel assisté}

Les parcelles en deuxième ou troisième rotation présentent des situations bien contrastées. Dans certaines parcelles, les arbres sont à forte densité, avec une croissance correcte, alors que dans d'autres les arbres sont clairsemés, très irréguliers et très branchus. Cela est peutêtre dû au savoir-faire de l'agrisylviculteur, ou à des problèmes de feu, d'épuisement du sol et d'érosion génétique des peuplements.

\section{Une base génétique des acacias trop étroite}

La plupart des acacias sont extrêmement branchus, y compris dans les plantations de première génération, en dehors d'une petite parcelle où les arbres sont très droits et monocaules (photo 8). Même en admettant que, pour la production de charbon, la forme n'a pas d'importance et qu'il convient d'optimiser la production de biomasse plutôt que celle de troncs rectilignes, il s'avère préférable de refaire de nouvelles introductions de matériel végétal, pour créer des peuplements semenciers à base génétique large, dont les descendants pourraient concilier forte production de biomasse et monocaulie (plus grande facilité de débit et de mise en tas, possibilité d'utilisation en perche et en petit sciage, en cas d'évolution du marché et de besoins locaux).

\section{Un projet de développement qui confirme les résultats de la recherche et qui ouvre des perspectives régionales}

Les plantations de Mampu sont très largement ignorées au plan international alors qu'elles constituent la seule vraie référence en Rdc (très peu de communication sur le projet). Pourtant, les résultats actuels du projet confirment sur une grande étendue (8 000 ha) les résultats obtenus de 1990 à 1994 par un projet de recherche européen à Oumé, en Côte d'Ivoire, sur la base des hypothèses des chercheurs (PELTIER et al., 1996 ; HARMAND et al., 2004). Ces résultats sont peu vulgarisés en milieu rural et ce système a été redécouvert à Mampu.

Aussi, il existe actuellement un regain d'intérêt pour les plantations multi-usages en Afrique centrale (MARIEn, MAllet, 2004). Plusieurs projets de plantations émergeront dans les années à venir, que ce soit au plan régional (Congo-Brazzaville) ou thématique (bois énergie, restauration de terres dégradées en zone périurbaine).

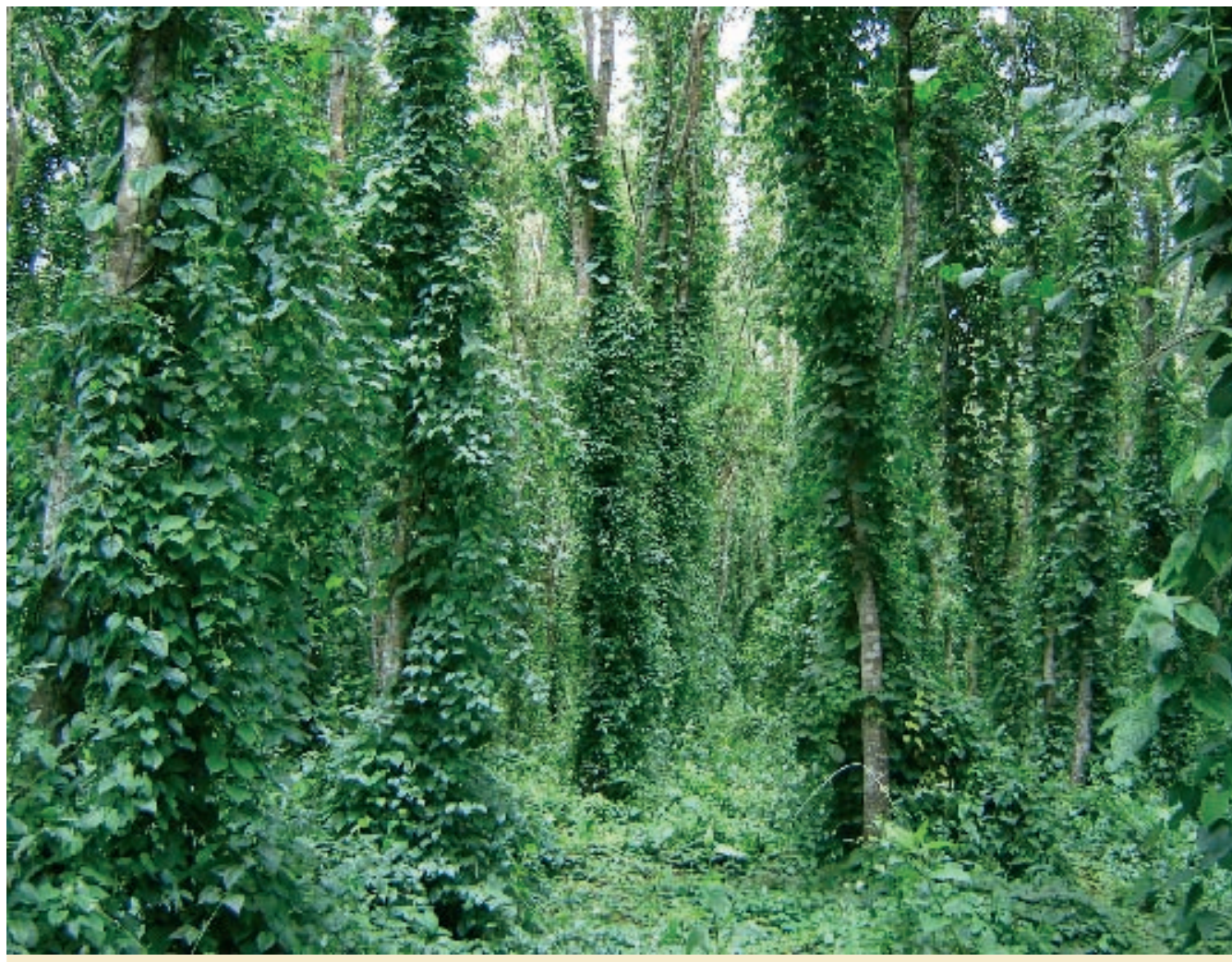

Photo 8.

Un écosystème forestier se reconstitue à l'abri des reboisements. Les ignames sauvages montent à l'assaut des acacias (ici une provenance monocaule). Photo R. Peltier. 

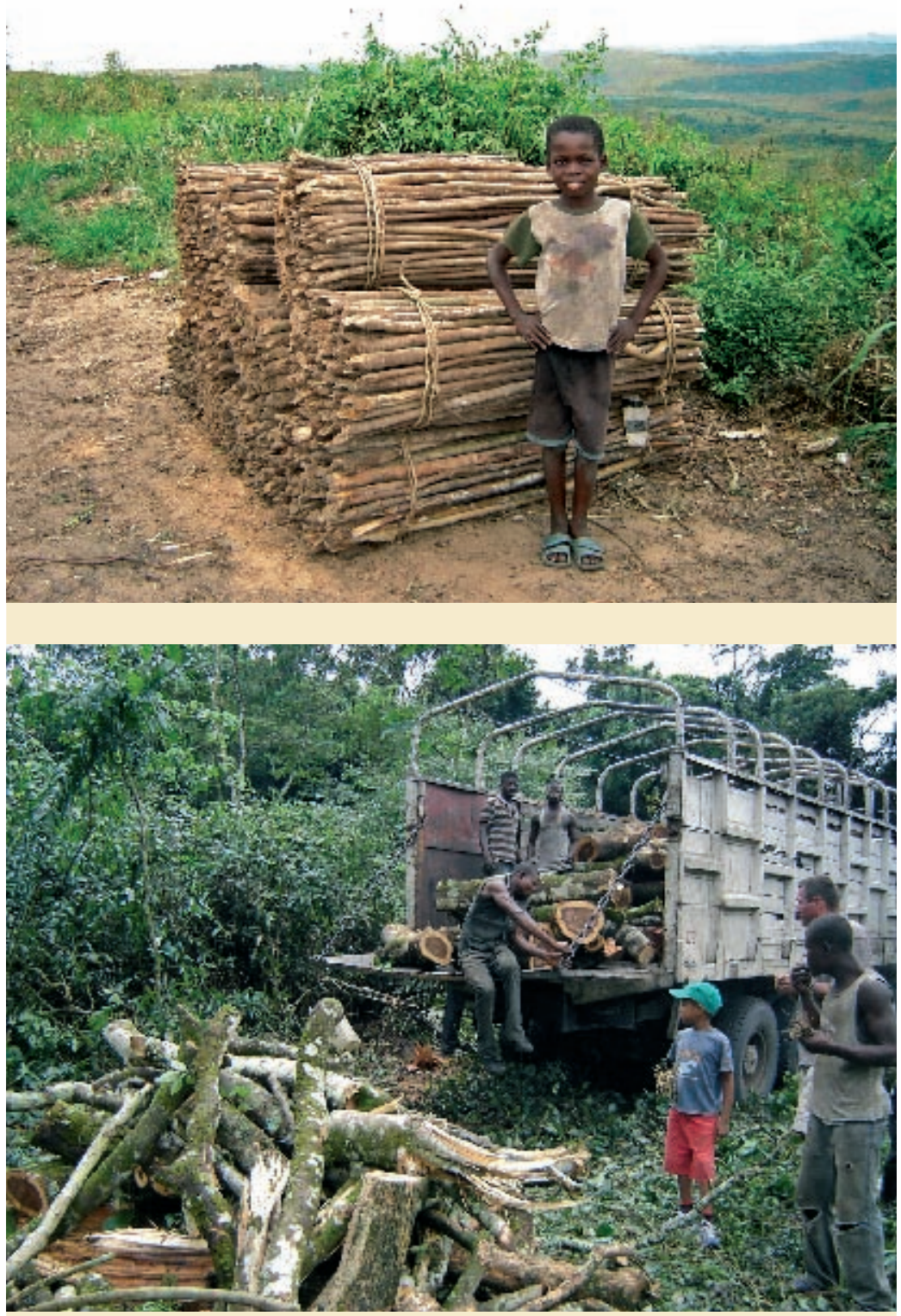

Photos 9.

Les fagots de perchettes de bois proviennent de la périphérie de la ville alors que les boulangers se fournissent directement en rondins de bois. Photo R. Peltier.

Enfin, la société privée Novace est à l'initiative récente de plantation d'acacias pour la fixation de carbone sur 400 ha début 2009, à proximité de Mampu (Ibi village). Elle utilise, quant à elle, les techniques du projet Mampu. La surface des plantations paysannes réalisées par les paysans eux-mêmes est de l'ordre de 6 ha par famille et l'objectif de plantation est de 1000 ha par an. Le total actuel des plantations dans cette zone approcherait 10000 ha.

\section{Mampu couvre une part importante des besoins de Kinshasa}

En comparant la production annuelle de charbon de Mampu (10 000 t) à la consommation théorique minimum de Kinshasa (600 000 t/an), le taux de 1,6\% peut sembler dérisoire. Considérant l'extrême pénurie d'énergie et la faiblesse des revenus de la majorité de la population, la consommation de
Kinshasa en charbon de bois est certainement surestimée. Une étude en cours sur le transport de charbon montre que, pour novembre et décembre 2008, Kinshasa aurait importé seulement 55000 sacs de charbon, dont 50000 sur l'axe est (aéroport-Mampu), l'axe sud-ouest Kinshasa-Mbanza-Ngungu étant très mal documenté à ce jour. Rapporté à l'année malgré un calcul approximatif, en raison des variations annuelles et de la non-prise en compte du transport de nuit, on obtient un chiffre de l'ordre de 20000 t/an de charbon. Ainsi des familles trouventelles des voies d'économie forcée, en ne préparant qu'un repas par jour et en bannissant les aliments à longue cuisson (haricots, etc.).

D'autres sources d'énergie existent, comme l'électricité produite par l'énorme barrage d'Inga, sur le fleuve Congo. Bien qu'il y ait sous-utilisation au regard des possibilités de production, les populations les plus aisés accèdent au réseau alors que les quartiers pauvres mal informés n'y accèdent que de façon informelle. Un survol nocturne de la ville met en évidence la présence du réseau électrique jusque dans les zones d'habitat spontané qui apparaissent éclairées aussi.

La sciure et les dosses de bois de scierie, les fagots de perchettes de bois provenant de la périphérie de la ville (photos 9) et divers déchets sont aussi récupérés. Plusieurs boulangers se fournissent directement en rondins de bois par leur propre circuit. Mampu assure de l'ordre de 5 à $10 \%$ de la couverture des besoins en charbon de bois de la ville, ce qui est remarquable sachant sa superficie $(0,3 \%$ de la surface du bassin d'approvisionnement de Kinshasa, demicercle de $150 \mathrm{~km}$ de rayon). 


\section{Autres modèles agroforestiers}

D’autres modèles de systèmes agroforestiers méritent d'être testés ou développés dans d'autres conditions écologiques et socio-économiques du pays, par exemple en gérant le recrû naturel d'espèces locales à usages multiples.

Le modèle de Mampu n'est pas à considérer comme l'unique modèle d'agroforesterie car il n'est pas applicable dans les conditions variées du pays. Le modèle Mampu est une réussite adaptée aux plateaux Batéké. Hélas, ce seul modèle de référence national s'avère inadapté dans des régions où le couvert forestier naturel existe; alors qu'une simple mise en défens suffirait pour retrouver une couverture végétale naturelle abondante.

Les populations Bakongo habitant les districts des Cataractes et de la Lukaya appliquent depuis des siècles le modèle des nkunku, un type de jachère améliorée ou assistée. On aboutit à la création de véritables agroforêts, avec dans certains cas protection continue sur une longue période. Les nkunku contiennent une grande diversité d'espèces locales (et parfois exotiques) qui y sont protégées et parfois réintroduites pour leurs multiples usages : production de fruits, feuilles d'emballage, feuilles consommées par les animaux domestiques, chenilles comestibles (LATHAM, 2003), support de champignons, fleurs butinées par les abeilles, pharmacopée, bois de feu et d'œuvre en éclaircie ou exploitation sanitaire. Le projet Makala étudiera ces agroforêts traditionnelles et déterminera les conditions techniques et socio-économiques de leur diffusion (encadré 1).

\section{Encadré 1. \\ Le projet Makala : une opportunité pour la diffusion des résultats du projet Mampu qui devrait, par ailleurs être renforcé par un projet de recherche.}

Lancé en février 2009, à l'occasion du séminaire de Kinshasa, le projet Makala, qui se propose de "Gérer durablement la ressource bois énergie en Rdc », est financé par l'Union européenne, pour une durée de quatre ans (MARIEN, 2009). Avec l'appui de nombreux partenaires congolais (dont le Snr pour les aspects reboisement) et internationaux, il se propose, entre autres, de tirer un bilan plus précis de l'expérience de Mampu (en partie sous forme de thèses de master ou de doctorat) et d'en promouvoir l'extension auprès de diverses sources de financement. Celle-ci pourra se faire soit sous sa forme actuelle de grands blocs sur des plateaux « vides », soit sous des formes beaucoup plus diffuses, dans l'espace rural approprié et régulièrement cultivé.

Un nouveau projet de recherche, en appui aux réalisations de Mampu (HARMAND, 2008) pourrait être formulé à travers les questions suivantes : - La carbonisation et l'exportation du charbon de bois, de même que le brûlis et la remise en culture, provoquent une perte de carbone et d'éléments minéraux et donc une baisse capitale de la fertilité acquise au cours de la phase de jachère. Cette perte est-elle préjudiciable au développement de la rotation suivante et donc au stockage de carbone à l'échelle du massif, ainsi qu'à l'efficacité de la nouvelle jachère dans la restauration de la fertilité du sol ?

- Quelle est l'influence de l'âge de la plantation en première rotation sur la production de biomasse et la restauration de fertilité ?

- À partir d'une chronoséquence de peuplements (de trois, quinze et vingt ans), quelles sont les dynamiques d'accumulation de matière sèche (biomasse) et d'éléments minéraux dans la jachère, de fixation d'azote, de production et de décomposition des litières aériennes, d'accumulation de carbone, d'azote et d'éléments minéraux dans le sol ?

- Quels indicateurs biologiques tels que la faune du sol et les microorganismes pourraient être mesurés dans ces systèmes et mis en relation avec l'âge de la jachère?

- Quelle est l'importance de l'amas de racines de la litière dans le fonctionnement du peuplement à partir de l'âge de cinq ans ?

- Quelle est l'influence de l'exploitation de la jachère sur la fertilité du milieu et le stockage de carbone?

En plus d'évaluer la quantité de nutriments exportée par la carbonisation, on pourrait estimer ce qui peut être restitué par les cendres de la carbonisation ainsi que la dynamique des stocks de carbone, d'azote et des éléments minéraux du sol (0-1 $\mathrm{m}$ de profondeur) et de la litière entre deux périodes clés : avant exploitation de la jachère et après abandon de la culture suivante. Un suivi dans le temps dans trois ou quatre parcelles pourrait être fait dans l'intervalle de dix-huit mois à deux ans. Une comparaison des stocks avec celui des savanes environnantes serait également utile. Enfin, un indicateur de fertilité serait la croissance de l'acacia en deuxième rotation en comparaison avec la première rotation. 


\section{Références bibliographiques}

BAUMER M., WOOD P., 1986. Agroforestry research and development : agroforestry practices for the solution of food, fodder and fuel shortages. In : Carlson L. W., Shea K. R. (éd.). Increasing productivity of multipurpose lands. Iufro research planning workshop for Africa Sahelian and North Sudanian zones, Nairobi, Kenya, 9-15 janvier 1986. Vienne, Autriche, lufro, 22-88.

BERNHARD-REVERSAT F., DIANGANA D., TSATSA M., 1993. Biomasse, minéralomasse et productivité en plantation d'Acacia mangium et $A$. auriculiformis au Congo. Bois et Forêts des Tropiques, 238 (4) : 35-44.

BERTRAND A., 1991. Étude des filières d'approvisionnement des villes en bois-énergie. Niamey, Niger, Projet Énergie II, volet Offre, Document technique $\mathrm{n}^{\circ} 11,57 \mathrm{p}$.

BRIANE D., DOAT J., 1985. Guide technique de la carbonisation. Aix-enProvence, France, Afme, Abf, Ctft. Edisud, $180 \mathrm{p}$.

COMBE J., BUDOWSKI G., 1979. Classification of agro-forestry techniques. In : De Las Salas G. (éd.). Agroforestry systems in Latin America. Proceedings of a workshop, Catie, mars 1979. Turrialba, Costa Rica, Catie, 17-47.

DUCENNE Q., 2009. Évaluation des actions agroforestières développées à Mampu, Rdc. Cardno Agrisystems Ltd. Volume I : Synthèse finale $32 \mathrm{p}$. Volume II : Rapport final 83 p.

DUVIGNEAUD P., 1949. Les savanes du Bas-Congo. Essai de phytosociologie topographique. Lejeunia, Revue Botanique, Mém. 10.

GAZULL L., 2009. Le bassin d'approvisionnement en bois-énergie de Bamako. Une approche par un modèle d'interaction spatiale. Thèse de doctorat, Université Paris 7 - Denis Diderot, France, $311 \mathrm{p}$.

GLASER B., LEHMANN J., ZECH W., 2002. Ameliorating physical and chemical properties of highly weathered soils in the tropics with charcoal : a review. Biology and Fertility of Soils, 35 : 219-230. HARMAND J.-M., 2008. Rapport de mission d'appui au Programme de réhabilitation de la recherche agricole et forestière, composante Recherche forestière, en République démocratique du Congo. Montpellier, France, Cirad, 10 p.
HARMAND J.-M., NJITI C. F., BERNHARDREVERSAT F., PUIG H., 2004. Aboveground and belowground biomass, productivity and nutrient accumulation in tree improved fallows in the dry tropics of Cameroon. Forest Ecology and Management, 188 : 249-265.

HARMAND J.-M., NJITI C. F., PELTIER R., 1997. Restauration de la fertilité des sols par la jachère arborée. In : L'agroforesterie pour un développement rural durable : recherche fondamentale et modélisation, applications tempérées et méditerranéennes. Atelier international, Montpellier, 23-29 juin 1997. Montpellier, France, Cirad, p. 135-142.

JENKINSON D. S., AYANABA A., 1977. Decomposition of carbon-14 labeled plant material under tropical conditions. Soil Science Society of America lournal, 41 : 912-915.

KOY KASANGO R., 2005. Impact of planted acacia forest on the chemical fertility of sandy soils of the Bateke Plateau (D.R. Congo). Msc. Thesis, Gent Universiteit (Université de Gand), Université libre de Bruxelles, Belgique, 121 p. + annexes.

LADMIRANT H., 1964. Carte géologique à l'échelle au 1/200 000 ; notice explicative de la feuille Léopoldville. Léopoldville, Congo, Service géologique du Congo.

LATHAM P., 2003. Edibles caterpillar and their food plants in Bas-Congo. Canterbury, Royaume-Uni, Mystole Publications, $60 \mathrm{p}$.

LEHMANN J., DA SILVA JR J. P., STEINER C., NEHLS T., ZECH W., GLASER B., 2003. Nutrient availability and leaching in an archaeological Anthrosol and a Ferralsol of the Central Amazon basin : Fertilizer, manure and charcoal amendments. Plant Soil, 249 : 343-357.

LEHMANN J., RONDON M., 2006. BioChar Soil Management on Highly Weathered Soils in the Humid Tropics. In : Uphoff N., Ball A. S., Fernandes E., Herren H., Husson O., Laing M., Palm C., Pretty J., Sanchez P., Sanginga N., Thies J. (éd.). Biological Approaches to Sustainable Soil Systems. Boca Raton, ÉtatsUnis, CRC Press (Book in Soils, Plants and the Environment, 113), p. 517-530. LUNDGREN B., 1987. ICRAF's first ten years. Agroforestry Systems, 5 : 197-217. MARIEN J.-N., 2009. Projet Makala, gérer durablement la ressource bois énergie en RDC. Projet EuropeAid DClENV/2008/151-384. Note de présentation, $7 \mathrm{p}$.
MARIEN J.-N., MALLET B., 2004. Nouvelles perspectives pour les plantations forestières en Afrique centrale. Bois et Forêts des Tropiques, 282 (4) : 67-79.

NAIR P. K. R., 1985. Classification of agroforestry systems. Agroforestry Systems, $3: 97-128$.

PELTIER R., 1993. Les jachères à composante ligneuse. Caractérisation, conditions de productivité, gestion. In : La jachère en Afrique de l'Ouest. Atelier international, Montpellier, 2-5 décembre 1991. Bondy, France, Orstom, p. 67-88.

PELTIER R., 1994. Du reboisement imposé à la prise en compte des savoirs traditionnels. Chroniques des sols Hardé du Nord-Cameroun. Natures Sciences Sociétés, 2 (1) : 67-79.

PELTIER R., BALLE PITY, 1993. De la culture itinérante sur brûlis au jardin agroforestier en passant par les jachères enrichies. Bois et Forêts des Tropiques, 235 (1) : 49-57.

PELTIER R., BALLE PITY, GALIANA A., GNAHOUA G. M., LEDUC B., MALLET B., OLIVER R., OUALOU K., SCHROTH G., 1996. Produire du bois énergie dans les jachères de zone guinéenne. Intérêts et limites à travers l'expérience d'Oumé en Basse Côte d'Ivoire. In : Fertilité du milieu et stratégies paysannes sous les tropiques humides : actes du séminaire, 13-17 novembre 1995. Montpellier, France, Cirad, p. 219-227.

ROBYNS W., 1948. Les Territoires phytogéographiques du Congo belge et du Rwanda Urundi. In : Atlas général du Congo belge. Publ. Ministère des Colonies, Bruxelles, Belgique.

TORQUEBIAU E., 1990. Introduction to the concepts of agroforestry. Nairobi, Kenya, Icraf, Working paper ${ }^{\circ}$ 59, 121 p. YOUNG A., 1988. Agroforestry and its potential to contribute to land development in the tropics. Journal of Biogeography, $15: 19-30$. 